\title{
Le rôle de l'adjectif dans la catégorisation des déchets
}

\author{
Jana Altmanova ${ }^{1}$, Claudio Grimaldi ${ }^{3}$, Silvia Domenica Zollo ${ }^{2}$ \\ 1,2,3 Università degli Studi di Napoli « L'Orientale », Dipartimento di Studi Letterari, Linguistici e \\ Comparati, via Duomo 219, 80138 Naples, Italie \\ ${ }^{1}$ jaltmanova@unior.it
}

\begin{abstract}
Résumé. Dans la présente contribution nous nous proposons d'examiner le statut de l'adjectif à valeur dénominative dans les procédés de catégorisation des déchets en contexte spécialisé et divulgatif. En analysant des séquences dénominatives du type déchet+Adj. extraites d'un corpus, nous allons observer la valeur sémantique et référentielle d'adjectifs relevant tous de la langue générale (ordinaire, classique, conventionnel, volumineux, encombrant(s), inerte(s), sauvage(s), solide) et qui, en entrant dans la composition du syntagme, semblent acquérir un statut semispécialisé, voire spécialisé surtout dans les typologies textuelles techniques. De même, nous allons analyser certains adjectifs de couleur (vert, marron, brun, blanc, gris, rouge, jaune), classés par Dixon (1977, 1982) parmi les huit classes d'adjectifs primaires, dont les propriétés sémantiques (expression du degré, fonction attributive, valeur descriptive) ne nous semblent pas toujours suffisantes pour expliquer leur emploi dans le contexte du classement des déchets.
\end{abstract}

\begin{abstract}
The role of the adjective in the categorisation of waste. In this paper we propose to examine the status of denominational adjectives in categorization processes of specialized and disclosing contexts. By the analysis of the déchet + Adj. structure extracted from the created corpus, the semantic and referential value of the adjectives accompanying the lexeme déchet will be examined. Many of these adjectives belong to the general language (ordinaire, classique, conventionnel, volumineux, encombrant(s), inerte(s), sauvage(s), solide) but, into the composition of déchet $+A d j$ structures, they acquire a semi-specialized status, even specialized, especially in technical textual typologies. Similarly, the analysis will take into account the massive presence of color adjectives (vert, marron, brun, blanc, gris, rouge, jaune), ranked by Dixon $(1977,1982)$ among the eight classes of primary adjectives, whose semantic properties (expression of the degree, attributive function, descriptive value) are often not sufficient to explain their use in the context of waste categorization.
\end{abstract}




\section{Introduction}

De nombreux travaux spécifiquement consacrés à l'adjectif ont désormais confirmé que ce dernier représente un élément capital dans la constitution du vocabulaire spécialisé. Dans la plupart des cas, un lien morpho-sémantique évident persiste entre l'adjectif et le nom qui le régit, alors que, dans d'autres cas, cette dépendance sémantico-référentielle apparait moins forte au moment constitutif du syntagme (L'Homme 1998, 2004 ; Maniez 2001a, 2001b, 2002 ; Frassi 2018). Ces déplacements de la référentialité des unités terminologiques semblent être déterminés aussi bien par la valeur sémantique de l'adjectif constituant que par la circulation des unités terminologiques en discours (Daille 2001 ; Lerat 2016).

De ce point de vue, le domaine de la gestion des déchets et de leur catégorisation, caractérisé dans les dernières décennies par une démultiplication terminologique constante, a été pour nous un domaine d'observation privilégié. En effet, la variété des produits à dénommer et la panoplie d'institutions impliquées concourent, sur le plan pragmatique, à déterminer un emploi élargi de cette terminologie par des experts et des non-experts du domaine (Galazzi, Desoutter 2017).

Dans cette contribution nous présentons les résultats d'une étude visant à explorer le statut des adjectifs primaires et non primaires dans la structuration de la terminologie des déchets et sa diffusion (Altmanova 2013), à travers l'analyse de quinze syntagmes terminologiques (ST) que nous avons sélectionnés sur la base de leur fréquence en discours, à partir d'un corpus expressément créé $(\S 2)$. Notre but est d'explorer le rôle référentiel de l'adjectif dans les ST retenus et d'en vérifier la valeur sémantico-pragmatique dans le discours inhérent à la gestion et à la catégorisation des déchets dont les dénominations sont susceptibles de suivre des transformations catégorielles en faveur de l'adjectif. Il nous semble que, malgré sa polysémie sémantique, celui-ci est - sur le plan pragmatique porteur du sens différentiel.

Premièrement, nous partons de l'hypothèse selon laquelle les adjectifs recueillis participent à l'enrichissement de la description attributive de l'univers référentiel concernant les différents types de déchets. En reprenant Kleiber (2001 : 25-26), nous pouvons donc affirmer que ces adjectifs participent à part entière à l'acte dénominatif, en exprimant une propriété référentielle qui catégorise le nom recteur déchet. Or, cette propriété n'est pas uniquement descriptive en termes de propriétés spécifiques (expression du degré, fonction attributive, valeur descriptive), car les adjectifs agissent en tant qu'opérateurs pragmatiques (Marengo 2012). Leur fonction principale est de créer une relation entre la dénotation du déchet et l'activité cognitive des locuteurs vis-à-vis d'un tel déchet.

Deuxièmement, d'une ultérieure réflexion il ressort que ces adjectifs, relevant de la langue commune, acquièrent dans ce contexte un statut semi-spécialisé voire spécialisé. Ces adjectifs ne constituent pas une catégorie grammaticale et terminologique discrète car ils se caractérisent par la graduation de leur sémantisme. En d'autres termes, ces adjectifs induisent les locuteurs à une lecture et interprétation ultérieure du référent, étant donné que dans l'acte de dénomination on assiste à un calcul interprétatif de la notion représentée par l'adjectif pour configurer cette dernière au regard du support nominal du terme même. Ces considérations nous conduisent à mener des réflexions qui portent notamment sur les dimensions sémantique et pragmatique à partir de deux catégories principales d'adjectifs que nous avons construites : 
- la première catégorie regroupe des adjectifs qui ont une signification plutôt transparente du point de vue référentiel et dénotatif. C'est le cas des adjectifs agricole, alimentaire, biodégradable, biomédical, radioactif, etc., dont la valeur attributive et descriptive apparaît suffisamment transparente et qui ne constituent donc pas l'objet de notre analyse ;

- la deuxième catégorie regroupe des adjectifs dont le sens est plus opaque et dont la qualité référentielle est moins intelligible, notamment en dehors du contexte juridico-administratif (à titre d'exemple, nous citons les adjectifs ordinaire, conventionnel, sauvage, inerte, rouge, gris, blanc, etc.).

La deuxième catégorie nous semble particulièrement intéressante de par la faible valeur référentielle des adjectifs, qui affecte la capacité de classer la réalité des déchets sur le plan linguistique. En d'autres termes, description et interprétation constituent les phases principales de la catégorisation des déchets qui peut être «objective» et transparente, lorsque le locuteur expert n'a pas recours à la dimension extralinguistique pour pouvoir interpréter et comprendre la réalité désignée ; "perceptive-subjective », quand les locuteurs font appel à des valeurs sémantiques implicites, dont l'usage peut aussi varier selon le contexte et selon des circonstances pragmatiques données. À la différence de quelques adjectifs que nous qualifions ici comme adjectifs à signification transparente, dont la classe dénotative a des contours stables ou relativement stables, celle des adjectifs à signification opaque semble être un ensemble flou (Kleiber 1979a, 1979b). Si certains sont classés selon les informations fournies par leurs traits sémantiques, d'autres sont en revanche plus difficiles à décrire.

\section{Ressources textuelles, terminologiques et documentaires}

Pour construire le matériel expérimental, nous avons utilisé différents types de ressources à partir desquelles nous avons confectionné des échantillons destinés à tester la méthodologie avant de l'étendre à des corpus et des ressources terminologiques plus conséquents. Le matériel que nous avons constitué se compose d'une sélection de termes techno-scientifiques provenant de l'utilisation simultanée de plusieurs bases de données et ressources lexicographiques: le Dictionnaire de l'Environnement ${ }^{1}$, le DicoEnviro Dictionnaire fondamental de l'Environnement ${ }^{2}$, le Grand Dictionnaire Terminologique $(G D T)^{3}$ de l'OQLF, EcoRessources ${ }^{4}$, FranceTerme ${ }^{5}$, TERMIUM Plus ${ }^{\circledR}{ }^{6}$, IATE $^{7}$ ainsi que Le Petit Robert $2017^{8}$ et le Trésor de la langue française informatisé (TLFi) ${ }^{9}$.

Ces premières tentatives d'évaluation de la productivité des procédés d'adjectivation menées à partir de bases de données et de dictionnaires, sont fortement sujettes à caution parce que celles-ci ne constituent pas des échantillons représentatifs d'une langue et surtout elles ne permettent pas de mesurer l'usage et la circulation des termes dans la langue, qu'elle soit générale ou spécialisée. Cette dernière exigence nous a demandé donc de recourir à des corpus au sens strict du terme, c'est-à-dire à des rassemblements de textes sélectionnés selon des critères qui garantissent la représentativité linguistique des éléments linguistiques explorés. Notre échantillon se constitue de 3400 occurrences totales de ST extraits :

- du corpus scientifique Scientext portant sur les sciences de l'environnement qui rassemble surtout des articles scientifiques ;

- d'un corpus de vulgarisation scientifique composé de documents et rubriques (Que faire de mes déchets?, Déchets scan et Bien jeter) pour les particuliers et éco-citoyens mis à disposition par l'Agence de l'environnement et de la maîtrise de l'énergie (ADEME) ${ }^{10}$, qui traite de sujets relatifs au traitement des déchets ; 
- d'un corpus composé de guides pratiques à la prévention des déchets mis à disposition sur le site France nature environnement $(F N E)^{11}$ et le site institutionnel consacré à la campagne nationale française de réduction des déchets Réduisons nos déchets ${ }^{12}$.

\section{Repérage et extraction des « candidats-adjectifs »}

Notre enquête a été réalisée en grande partie de manière manuelle, alors que l'extraction des termes a fait l'objet d'une procédure automatique avec annotation humaine. Un extracteur en libre accès a été utilisé, TermoStat (Drouin 2003), ce qui nous a permis d'augmenter le nombre d'adjectifs et d'améliorer aussi la qualité des candidats-termes complexes. Nous avons donc lancé la recherche en sélectionnant le paramètre «termes complexes nominaux ». À partir de la liste des candidats-termes élaborée par l'outil, nous avons fait une évaluation manuelle par annotation afin de valider tout d'abord la validité syntagmatique des termes-candidats complexes, leur appartenance au domaine des déchets et leur emploi en tant que termes dans le contexte d'apparition. Ensuite, nous avons procédé progressivement à la sélection des adjectifs qui font l'objet d'un emploi terminologicoréférentiel sur la base de deux critères : l'un morpho-syntaxique, l'autre sémantique. Dans le premier cas, nous avons retenu des séquences $\mathrm{N}_{-}$Adj. ; dans le deuxième cas, nous nous sommes concentrés sur le rapport entre le nom et le pseudo-adjectif «classif[iant] et catégoris[ant] les noms qui, à leur tour, classifient (ou instituent) un monde d'objets (les référents) ou de phénomènes présupposés réels ou existants [...]» (Bartning 1980 : 70).

En suivant cette démarche, nous avons isolé dans notre corpus, à titre d'exemple, quinze syntagmes terminologiques à savoir déchet ordinaire (103), déchet classique (211), déchet conventionnel (235), déchet volumineux (456), déchets encombrants (500), déchet inerte(s) (465), déchet sauvage (433), déchet solide (787), déchet vert (899), déchet brun (601), déchet marron (568), déchet gris (345), déchet blanc (221), déchet jaune (356) et déchet rouge (231). Cette première étape de travail nous a permis d'obtenir des résultats satisfaisants quant à l'approche suivie qui, nous semble-t-il, peut ajouter quelques réflexions utiles et préalables à la description de l'usage des adjectifs primaires et non primaires (Dixon 1977, 1982) dans la terminologie du traitement et de la gestion des déchets.

\section{Les adjectifs classifiants}

Afin de mieux cerner le phénomène de l'ambigüité référentielle, nous voudrions faire une brève précision d'ordre terminologique sur ce que nous entendons pour ce concept (Kleiber 1979). Nous considérons ambigus d'un point de vue référentiel tous les adjectifs qui désignent des éléments difficilement intelligibles à cause des facteurs extralinguistiques, pragmatiques et perceptifs qui induisent les locuteurs à une lecture et une interprétation ultérieures du référent. Parmi les syntagmes terminologiques repérés dans notre corpus, certains d'entre eux permettent de réfléchir de manière privilégiée sur le mode de fonctionnement de la prédication adjectivale, étant donné que dans l'acte de dénomination on assiste à un calcul interprétatif de la notion représentée par l'adjectif pour configurer cette dernière par rapport support nominal du terme même. Le trait qui caractérise les ST de ce groupe est que les adjectifs, tous relevant de la langue générale, impliquent une référence qui s'exprime de plusieurs manières sur une échelle de degrés, ou bien une série, une suite continue et progressive de niveaux linguistiques qualifiants et 
typologisants qui constituent une véritable hiérarchie dans le classement terminologique des syntagmes.

Commençons par analyser quelques séquences déchet_Adj situées sur le premier point d'une échelle de degrés d'opacité jusqu'à arriver aux ST moins opaques où, d'après nous, trois facteurs peuvent contribuer à l'intuition du degré de transparence/opacité de ces adjectifs (la métaphore conceptuelle, le sens sémantique et la conventionalité du sens).

Le premier cas concerne l'adjectif ordinaire ayant dans la langue générale deux sens : celui de la conformité à l'ordre normal et habituel des choses sans conditions particulières et celui faisant référence à la qualité qui ne dépasse pas le niveau moyen de la normalité et qui n'a aucun caractère spécial $(P R)$. Au contraire, dans la catégorisation des déchets, il renvoie à d'autres valeurs et à d'autres propriétés. Comme l'indique le GDT, le déchet ordinaire désigne un « déchet provenant d'un site nucléaire, pris en charge par les filières d'élimination soit des déchets ménagers ou assimilés, soit des déchets industriels ». Dans cette définition, l'extension logique du concept ordinaire renvoie à une caractéristique techno-administrative, à savoir la manière habituelle de traiter ces déchets, qui implique une certaine culture de la part des destinataires afin d'éviter de confondre sens général et sens technique. On s'approche ici de ce que Lerat appelle «définitions technoadministratives » (Lerat 2016: 85), c'est-à-dire des définitions stipulatives. En effet, par rapport au sens premier de l'adjectif ordinaire, nous remarquons une certaine opacité référentielle représentée par l'absence de traits sémantiques liés à l'adjectif ordinaire qui, dans ce cas, semble spécifier plutôt la modalité de prise en charge dans la filière d'élimination que la nature des déchets impliqués. Dans le langage professionnel, cette typologie de déchet est parfois dénommée déchet classique, dénomination recommandée depuis 2009 par la Commission d'enrichissement de la langue française. Comme pour ordinaire, l'adjectif classique fait ici référence implicitement à la manière usuelle de traiter ces déchets et non pas au processus d'identification du déchet.

Un autre adjectif a attiré notre attention : il s'agit de l'adjectif conventionnel qui dans la séquence déchet_Adj désigne, d'après le GDT, un « déchet non radioactif provenant d'une installation nucléaire de base et qui, à ce titre, fait l'objet d'une gestion spécifique ». Comme pour les deux cas précédents, l'adjectif conventionnel, qui qualifie un référent faisant l'objet d'un accord entre deux ou plusieurs parties, se réfère à la gestion de ce produit et non pas à la nature du déchet même. En s'agissant d'un déchet non radioactif, mais qui provient d'une installation nucléaire, il est traité spécifiquement, suite à une convention, un accord, raison pour laquelle il est qualifié de conventionnel.

Dans les trois séquences analysées, la catégorisation des déchets se réalise grâce aux adjectifs classifiants qui renvoient au traitement spécifique auquel les résidus, provenant d'un site nucléaire, sont soumis. Ces adjectifs peuvent être considérés comme catégorématiques, dans la mesure où une partie de leur sens dépend du porteur qu'il qualifie. Toutefois, il nous semble qu'une adaptation du contenu adjectival au contenu du nom a lieu par l'incorporation de notions supplémentaires qui nécessitent une réflexion et une interprétation pour rendre explicite la dilatation au niveau sémantique des adjectifs pouvant être indiqués comme constructeurs de la référence.

Un cas très intéressant est représenté par l'adjectif volumineux ainsi défini par le TLFi: «qui développe, présente des proportions importantes, un volume supérieur à la moyenne ; qui occupe beaucoup d'espace ». Il est important de signaler qu'il n'appartient pas spécifiquement à la terminologie de la gestion des déchets, étant donné que son emploi caractérise autant la langue commune que la langue de spécialité. Seuls le GDT et la base de données EcoRessources le situent à l'intérieur du domaine des déchets, en cooccurrence avec le substantif déchet, en proposant la définition suivante : « déchets qui ne peuvent être 
éliminés avec les ordures ménagères courantes en raison de leurs dimensions ou de leur poids excessifs ». Le transfert métaphorique du trait sémantique de l'adjectif volumineux, à savoir celui de la grande taille, du volume et des proportions importantes, permet de catégoriser de manière référentielle, bien que par approximation, une typologie de déchets et de la distinguer des autres par la dimension et le poids qui sont nettement supérieurs aux autres déchets. De fait, l'unité terminologique déchets volumineux englobe les réfrigérateurs, les fours, les matelas, les canapés, les portes et certains appareils électroniques.

Par le même procédé de création lexicale est construit aussi le synonyme déchets encombrants faisant référence au même noyau sémique ainsi que l'adjectif dénominal encombrants, dont l'emploi substantivé au pluriel encombrants est signalé par le $G D T^{13}$.

Le même phénomène peut être relevé dans le ST déchet inerte qui est répertorié par le Dictionnaire de l'Environnement et le GDT. Ceux-ci en proposent les définitions suivantes : " [déchet inerte] désigne un déchet qui ne subit aucune modification physique, chimique ou biologique ${ }^{14}$. Dans ce cas aussi, nous relevons une catégorisation adjectivale par métaphore terminologique. En effet, le trait sémique qui fait l'objet d'une extension de sens est celui de « inactif», « qui n'a plus d'activité » (TLFi), ce qui est d'ailleurs souligné par le GDT. De par leurs caractéristiques, les déchets inertes sont inactifs au sol et n'entraînent aucune réaction chimique ou toxique sur les sols ou dans les airs. D'où le transfert des deux traits distinctifs : le premier concernant l'état «inactif», le second le résultat « sans réactions ».

Dans le domaine des déchets, l'adjectif inerte a produit des dérivés comme le substantif inerte, employé notamment au pluriel inertes, pour désigner cette catégorie de déchets issus de la terre, tels que carrelage, briques, ciment, cailloux, gravat, verre à vitre, porcelaine $^{15}$, comme dans l'exemple suivant :

1) Un premier contrôle visuel sera effectué dans la benne lors de l'entrée du site afin de vérifier la conformité des inertes et un enregistrement détaillant le type de matériau, le nom de l'apporteur et le lieu en cas de chantier interne au groupe, sera effectué en amont (http://www.drome.gouv.fr/IMG/pdf/ISDI_Alixan_signe_21-30.pdf).

De même, la forme verbale inerter apparaît dans notre corpus (331 occurrences), pour indiquer l'action de rendre inactifs les déchets, comme l'atteste l'exemple suivant:

2) D'autres procédés visant à inerter les déchets toxiques sont possibles ou en cours d'expérimentation. (https://www.senat.fr/rap/o98-415/o9841516.html)

Le ST déchets inertes a donc donné naissance à l'adjectif à valeur substantivale inertes par un processus d'abréviation et de réduction lexicale répondant à un besoin communicationnel spécialisé basé sur le principe de l'économie linguistique (Kocourek 1982 : 141 ; Lerat 2016 : 105). Cela est possible parce que la charge sémantique se déplace sur l'adjectif, au fur et à mesure que le nom déchet s'évide de sens. Quant au verbe inerter, celui-ci contient ici le trait sémique de l'inactivité, ou bien celui de rendre inactifs les déchets toxiques et de leur apporter cette caractéristique propre aux inertes. Outre le processus intuitif de la métaphorisation, nous assistons aussi à un procédé de changement de catégorie grammaticale produisant deux néologismes sémantiques créés à partir du mécanisme de conversion syntaxique (Adj. $\rightarrow$ Nom ; Adj. $\rightarrow$ Verbe) (Assal 1994). 
Parmi les autres syntagmes de notre échantillon nous signalons aussi quelques phénomènes de création par analogie. C'est le cas du syntagme déchet solide qui est répertorié aussi au pluriel dans le GDT et dans la base de données EcoRessources pour désigner «tout produit résiduaire solide à $20^{\circ} \mathrm{C}$ provenant d'activités industrielles, commerciales ou agricoles, détritus, résidu d'incinération, ordure ménagère, gravats, plâtras et autre rebut solide à $20^{\circ} \mathrm{C}$ ». Il est ainsi appelé par analogie avec la dureté et la consistance physique des résidus, exception faite pour les «carcasses de véhicules automobiles, des terres et sables imbibés d'hydrocarbures ».

Dans les ST déchet_Adj. repérés dans le corpus, d'autres adjectifs relevant de la langue commune sont impliqués dans des procédés de métaphorisation dont le résultat final est la création d'une sous-catégorie de déchets plus spécifiquement employée dans des contextes spécialisés tel que sauvage. Comme nous pouvons le remarquer dans le TLFi, l'adjectif sauvage est très polysémique, ayant acquis au cours des siècles plusieurs sens selon le référent désigné par l'adjectif même. Dérivé du latin classique selvaticus (dérivé de silva «forêt ») indiquant ce «qui est fait pour le bois » et puis employé à l'époque impériale pour parler d'une plante, cet adjectif a survécu dans les langues romanes pour évoquer un objet, un artefact, un individu ou une chose conforme à l'état naturel et qui n'a pas subi l'action de l'homme. Le trait sémantique qui a été transféré dans la séquence déchet sauvage est celui de «[en parlant d'une chose] qui échappe aux règles établies, qui se fait en dehors de toute organisation officielle, qui a un caractère spontané et incontrôlable ». Par métaphorisation, il représente les détritus et les immondices qui se trouvent dispersés sur le sol dans les milieux naturels et comprend plus précisément les objets hétéroclites, les boîtes à pique-nique, les vieux journaux, les bouteilles cassées, les boîtes à conserves, les vieux bidons, les matelas éventrés, etc.

En ce qui concerne les sources lexicographiques consultées, déchet sauvage n'est pas enregistré dans le Dictionnaire de l'Environnement qui, par contre, propose 8 occurrences de l'adjectif faisant référence à la faune sauvage et aux décharges et dépôts sauvages. De même, le Dictionnaire fondamental de l'environnement offre un seul résultat concernant le domaine de la faune et des espèces menacées, alors qu'aucune définition n'y est fournie.

Nous relevons une définition de l'adjectif sauvage postposé au nom déchet dans le Grand Dictionnaire Terminologique (GDT) qui propose le ST uniquement au pluriel (déchets sauvages) datant de 1987 :

Déchets échappant aux circuits habituels de collecte et d'élimination et se retrouvant de façon dispersée sur le sol, le plus souvent dans le milieu naturel (plage, forêt, bord des routes et des cours d'eau, etc.).

Dans ce domaine, l'adjectif sauvage a donné vie, par le procédé de la métaphorisation, à d'autres collocations formées sur le même modèle que le syntagme déchet sauvage, tels que combustion sauvage, épandage sauvage, décharge sauvage. À ce propos, nous croyons nécessaire de nous arrêter en particulier sur l'usage du ST décharge sauvage, normalisé en 1983 par l'Association française de normalisation (AFNOR) et ainsi défini par le GDT:

Décharge qui reçoit des déchets de toute nature dans des conditions qui ne respectent pas les règles de la décharge contrôlée.

Note. Lorsque ces déchets proviennent de la commune, la décharge est dite «brute » et, dans les autres cas, « sauvage ». 
Ici l'adjectif sauvage catégorise en quelque sorte une typologie de décharge, désignant un endroit illégal où les gens laissent leurs déchets, sans aucune autorisation et qui se différencie de la décharge contrôlée. Bien que le sens du ST soit saisissable, il ne l'est que partiellement, car sa définition pragmatique et techno-administrative, échappe à tout nonexpert du domaine. Dans leur fonction référentielle-dénotative, les adjectifs semblent donc participer à l'extension de la catégorie des déchets qui peuple l'univers référentiel, en se basant sur l'emploi attributif (catégorisation sémantique) mais aussi sur l'emploi contextuel (inscription référentielle pragmatique) qui peut se vérifier dans les interactions verbales lorsque les locuteurs mettent en jeu un ensemble de connaissances encyclopédiques et sociales.

\section{Les adjectifs de couleur}

L'exploitation du corpus créé nous a permis de confirmer l'hypothèse selon laquelle les adjectifs de couleur (AdjCoul) sont massivement utilisés dans la catégorisation aussi bien des déchets que des conteneurs de déchets soumis au tri sélectif. Dans la lignée des réflexions proposées précédemment, nous allons démontrer comment ces adjectifs participent à part entière à l'acte dénominatif, en exprimant une propriété référentielle qui catégorise la base déchet. En effet, comme le démontre Gross (1996 : 42), les AdjCoul ne sont pas toujours sources de prédiction et n'ont pas vraiment le sens habituel qu'il est possible de relever dans d'autres contextes, étant donné qu'ils se prêtent rarement à la description. D'ailleurs, bien qu'elle puisse être appréhendée dans son acception métonymique, la couleur n'est pas considérée comme une propriété physique objectivement observable. Comme l'affirme Bosredon (1991 : 98) pour sac jaune:

[...] un ensemble de «sacs jaunes » et même tous les sacs jaunes du monde depuis que le monde existe ne formeront jamais un ensemble d'éléments dont je pourrai dire dans un accès de fièvre logiciste : sac jaune est le nom d'un sac dont la couleur est jaune contenant qui est tel et tel autrement dit sac jaune.

En ce qui concerne les dénominations des déchets, les AdjCoul détectés dans notre corpus se prêtent - au moins dans une première phase de nomination - presque uniquement au processus de référenciation que nous allons mettre en évidence à travers les propriétés sémantiques et pragmatiques (facteurs extra-linguistiques) des AdjCoul mêmes. Dans le processus de référenciation, un certain nombre de structures correspondent donc à des structures dénominatives. Nous analyserons par la suite quelques exemples d'AdjCoul référentiels présents dans notre corpus (vert, brun, marron, blanc, gris, rouge, jaune) qui permettent de construire une classe référentielle partiellement reconnue, en opérant une sous-catégorisation. En effet, bien qu'ils possèdent les propriétés syntaxiques et sémantiques des adjectifs qualificatifs, dans le domaine environnemental ils s'avèrent être référentiellement sous-catégorisants, ce qui implique un autre type d'analyse. Comme le souligne, d'ailleurs, Gross (1996 : 50) pour la catégorisation des vins :

Dans les suites vin blanc, vin rouge et vin gris les adjectifs ne sont pas des prédicats ; ils ne permettent pas une véritable assertion. L'adjectif n'a pas pour but d'apporter au substantif vin une propriété physique donnée (sa couleur) mais de désigner des types de vin différents, des variétés de vin [...]. 
On observera, au passage, que du point de vue de la couleur objective, le vin blanc est jaune et le vin gris rosé.

En d'autres termes, la combinaison du substantif et de l'adjectif induit à une interprétation sous-catégorisante du syntagme et la couleur en tant qu'élément chromatique de description est reléguée ici à un second plan (Rodríguez Pedreira : 27).

Le premier syntagme terminologique dont nous proposons l'analyse dans le contexte de la gestion des déchets est représenté par l'unité dénominative déchet vert, très fréquente dans le corpus créé, notamment dans les textes de nature principalement divulgatrice, dont nous avons relevé deux définitions dans le Dictionnaire de l'Environnement ${ }^{16}$ et dans le $G D T^{17}$ :

Un déchet vert (DV) désigne un déchet végétal résultant de l'entretien et du renouvellement des espaces verts publics et privés (parcs et jardins, terrains de sports, etc.), des collectivités territoriales, des organismes publics et parapublics, des sociétés privées et des particuliers. Du point de vue réglementaire leur collecte est régie par le décret du 7 février 1977, relatif à la collecte des déchets encombrants, leur élimination par la loi cadre du 15 juillet 1975. Le brûlage est interdit, ainsi que le dépôt sauvage, pouvant entraîner une pollution des sols. Une des voies de valorisation les plus intéressantes reste le compostage sur plate-forme, pouvant relever de la réglementation sur les installations classées (ICPE).

Déchet d'origine végétale résultant de la taille, de l'entretien ou de l'exploitation par l'homme d'un milieu végétalisé.

Note : Issus d'espaces publics ou privés, de jardins ou du milieu forestier, les déchets verts sont composés de feuilles, de branches, d'écorces, de résidus de tonte de gazon, de fleurs fanées, de copeaux de bois, de racines, etc. Ils sont en général compostables.

Comme nous pouvons le remarquer dans la première définition, il semblerait que la dénomination déchet vert soit déjà utilisée en 1977 lors de la parution du décret relatif à la collecte des déchets encombrants, pour se référer aux déchets végétaux provenant des espaces verts (parcs, jardins, terrains de sports, etc.). Dans le discours environnemental, l'AdjCoul vert est employé pour faire référence à toute forme d'action écologique et de protection de la nature. Par synecdoque, le vert, qui est la couleur de la nature ou d'une partie de la nature, indique la nature elle-même. Dans le cas des déchets, par un glissement de sens de type métonymique, il désigne les déchets dérivés des espaces naturels qui sont compostables, d'où la présence du sème de la protection de la nature et de sa valorisation. Si l'efficacité de la couleur verte pour représenter l'aspect écologique peut se comprendre grâce au contexte historique et réglementaire, il semblerait que la raison de la lexicalisation de cet AdjCoul nécessite aussi une réflexion de type morphosémantique.

Nous retrouvons une entrée de l'AdjCoul vert dans Termium accompagné de nombreux synonymes en langue française et en langue anglaise. Les synonymes, qui sont des lexèmes comportant les sèmes de la nature et de la protection de l'environnement, sont écologique et respectueux de l'environnement alors qu'en anglais l'adjectif green apparaît de même que d'autres adjectifs tels que ecological, eco-friendly, environmentally friendly, earth-friendly et respectful of the environment. Cependant, il est évident que l'AdjCoul vert et son équivalent anglais green sont les plus utilisés pour sous-catégoriser cette classe de déchets 
car c'est sur la base d'une propriété reconnue comme intrinsèque, ou bien celle du respect de la nature et de la non pollution, que l'on choisit l'adjectif à associer à déchet dans le syntagme (Rakotonoelina 2017) et cela aussi bien dans la langue spécialisée que dans le discours de vulgarisation. Nous pouvons donc affirmer que dans le cas de déchert vert l'AdjCoul n'a aucune valeur descriptive, mais il renvoie à une série de références culturelles partagées, à savoir des connaissances autres que linguistiques.

Un autre type de couleur est lié à la façon de représenter cette typologie de déchet: l'adjectif brun renvoie à « une couleur qui rappelle celle des feuilles mortes » (TLFi), et, bien qu'il n'apparaisse pas dans les ressources lexicographiques et terminographiques consultées, il est présent dans le corpus textuel de référence, notamment dans les textes concernant l'emploi des déchets provenant des jardins privés et d'autres espaces verts. En voici un exemple :

3) Pour réussir votre compost, mélangez les déchets de catégories opposées : les déchets verts avec les déchets bruns, les matières humides (gazon, déchets de cuisine...) avec les matières sèches (papier, branches...) et les déchets grossiers avec les plus fins (http://www.ccpays-

solesmois.fr/fileadmin/user_upload/Dechets/Petit_guide_du_compost_B oreal.pdf).

Sur le plan référentiel, le syntagme terminologique déchet brun est lié à celui de déchet vert. Il désigne principalement des matières carbonées, dures et sèches, utilisées dans le compostage, comme les branches, les feuilles mortes, la paille, le foin sec, les fleurs coupées et les feuilles d'automne qui se caractérisent par une même nuance de couleur qui tend au marron. Toutefois, si comme pour vert, l'AdjCoul brun semble renvoyer, d'une part, à l'aspect extérieur des éléments végétaux utilisés dans le compostage, d'autre part, d'ultérieures sources textuelles consultées comptent parmi les déchets bruns des coquilles d'œufs et leur emballage, sachets de thé, carton, papier essuie-tout, serviettes en papier, ce qui empêche d'attribuer à l'AdjCoul brun une valeur strictement descriptive et qualitative :

4) La variété des apports est indispensable à la bonne conduite de votre composteur. Il faut alimenter le bac en matières vertes et matières brunes. Matières vertes : déchets qui retiennent l'humidité (tonte de gazon, épluchures, reste de repas). On parle également de déchets humides ou azotés. Matières brunes : déchets secs ou carbonés qui aident l'eau en excès à mieux s'écouler (carton ondulé, boîte à œufs, broyat). Il est nécessaire d'alterner les apports verts/bruns pour que le compost soit équilibré et que la décomposition se fasse de manière optimale (https://www.agglo-seine-eure.fr/attachment/811382/ Guide $d u$ compostage et du jardinage au naturel, Communauté d'agglomération Seine - Eure, Décembre 2015).

En prenant en compte la production du compost en tant que produit final de l'utilisation des déchets bruns - syntagme coexistant avec déchet marron qui est toutefois moins lexicalisé et employé au niveau discursif -, l'AdjCoul brun semble désigner plutôt le compost en tant que produit stabilisé semblable à un terreau, riche en composés humiques que les résidus qui forment le compost. En comparant les ST déchet vert et déchet brun, les deux adjectifs de couleur vert et brun sont partiellement liés à la couleur des référents 
insérés dans la catégorie des déchets verts (Rakotonoelina 2017 : 22-24) et déchets bruns, le chromatisme étant placé en second plan car l'identification du déchet ne dépend pas de la couleur qu'il représente. L'adjectif caractérise donc le nom en lui conférant une couleur et toute une série de connaissances extralinguistiques, grâce auxquelles l'occurrence peut être identifiée comme appartenant à une classe donnée. Dans ce système de classification, les AdjCoul créent donc des sous-catégories, ce qui s'explique de manière générale par le fait que les couleurs sont étroitement corrélées à la nature des notions impliquées.

Dans la catégorie des déchets d'équipements électriques et électroniques (DEEE, D3E ou PEEFV-produits électriques et électroniques en fin de vie), dont la Directive 2002/96/CE vise à rendre obligatoire la valorisation, sont inclus les déchets bruns, les déchets blancs et les déchets gris, bien que la Directive citée n'utilise pas directement l'indication de ce type de dénominations. La création du sous-classement répond ici de manière évidente à la nécessité de séparation des différentes typologies d'équipements électriques et électroniques inclus parmi les DEEE :

5) On classe les DEEE en trois catégories : les déchets blancs (électroménager), les déchets gris (informatique, téléphonie, bureautique) et les déchets bruns (audiovisuel). La production de déchets a suivi l'extraordinaire envolée des ventes de ces matériels pendant ces dernières décennies. On ne traitera ici que l'aspect ordures ménagères mais il faut savoir que les déchets gris sont aussi méthanisés au stade industriel (www.ecosociosystemes.fr/traitement_dechets.html).

Dans le Dictionnaire de l'Environnement nous avons relevé ces AdjCoul dans la définition de monstres, terme qui sert à désigner les gros électroménagers, bien que, si l'on tient compte de la définition précédente, ce ne soient que les déchets blancs qu'il faudrait indiquer en tant que produits électroménagers :

Désigne les déchets des ménages volumineux (encombrants sans les déchets verts et les gravats) : mobilier, gros électroménagers (blanc, brun et gris), ferraille (deux roues, grillage, etc.) et autres objets usagés.

Un déchet blanc est un déchet issu des habitations et concerne les produits électriques, tels que frigos, machines à laver, cuisinières, appareils de lavage et de cuisson, réfrigérateurs, appareils de chauffage et d'autres équipements électriques divers. En effet, ceux-ci sont généralement de couleur blanche qui dans la dénomination déchet blanc exprime métonymiquement l'aspect extérieur et physique des déchets :

6) Les déchets blancs (frigo, congélateurs, etc.) font l'objet d'un chargement en benne et d'un pré-tri au déchargement pour permettre un reconditionnement et un recyclage (déchets D3E) (http://www.tdslocation.com/Transports-TDS-une-expertise-dans-denombreux-domaines_348.html).

7) Cette information sur le coût du recyclage, qui sera clairement affichée pour le consommateur, est prévue pendant huit ou neuf ans pour les déchets blancs (réfrigérateurs), et sept ans sur les bruns (télévision, ordinateur) 
(https://www.caissedesdepotsdesterritoires.fr/cs/ContentServer?pagenam $\mathrm{e}=$ Mairie-conseils/MCExperience/Experience\&cid=1245645192002).

Quant au ST déchets gris, celui-ci désigne les déchets de l'informatique, de la téléphonie ou de la bureautique (les ordinateurs, les imprimantes, les scanners, ainsi que les photocopieuses, les téléphones ou répondeurs) :

8) Gris (produit - ou déchet -) : produits provenant de l'informatique, de la bureautique, des télécoms. Voir aussi Poubelle grise (Guide $d u$ traitement des déchets, $2013:$ 438).

Il est intéressant de remarquer que parmi les DEEE, les sources consultées - qui d'ailleurs ne s'avèrent pas être des textes que l'on pourrait indiquer comme textes de vulgarisation - citent aussi les déchets bruns, à savoir le matériel audiovisuel, les postes de radio et de télévision, les caméscopes et magnétoscopes, les chaînes hi-fi, les instruments de musique, ce qui nous permet d'avancer l'hypothèse d'une certaine confusion sur le plan de la désignation. Le même ST désigne, tel qu'on l'a vu, des résidus tout à fait différents, aussi bien pour leur nature que pour leur utilisation dans la gestion des déchets. Pour les DEEE l'emploi de l'épithète de couleur ne semble correspondre que partiellement à des raisons de qualification des déchets par le biais de leurs caractéristiques extérieures, déchet blanc étant, de ce point de vue, le seule ST dans lequel la couleur blanche renvoie de manière plus ou moins évidente au référent désigné. Toutefois, dans ce cas aussi, il nous semble que la valeur descriptive de l'AdjCoul est toujours faible car l'identification du déchet en question repose principalement sur sa nature de déchet électroménager plutôt que sur sa couleur. Quant à déchet gris et déchet brun, ce dernier étant considéré comme DEEE, les motivations sont encore plus faibles (la couleur grise pourrait renvoyer à l'image prototypique des appareils informatiques) et le choix de l'épithète servant de moyen de sous-catégorisation semble plutôt flou. Cela confirmerait notre hypothèse selon laquelle si en général la référence des structures nominales s'opère au niveau de la couleur, ce n'est pas toujours le cas. Il y a aussi des propriétés et des facteurs extra-linguistiques qui favorisent le processus de sous-catégorisation. Comme l'affirme Leduc-Adine (1980:83) :

Si la couleur constitue une qualité inhérente, liée à la substance des choses elle n'a pas vraiment de contenu propre, pas de sens particulier, elle n'est que cernée, que saisie, et sa première propriété est de renvoyer à quelque chose d'autre qu'elle-même, qui est plus important qu'elle : ainsi un lien profond s'établit entre la couleur et la vie.

À notre avis, un certain degré d'arbitraire du choix des AdjCoul dans le domaine des déchets est encore plus évident en ce qui concerne les dénominations des bacs pour le recyclage. Prenons, en guise d'exemple, les dénominations des bacs pour le recyclage bac jaune, bac vert, bac rouge, bac noir, bac marron. Alors que c'est strictement la couleur qui implique la référenciation dans le cas de bac vert pour les bouteilles (renvoyant à l'image prototypique du récipient) et rouge pour les déchets ne pouvant pas être recyclés (le rouge est la couleur du danger et des codes d'alerte), relativement à bac bleu pour les journaux, annuaires, magazines, etc. ou le jaune pour les métaux, flacons, emballages plastiques, la sous-catégorisation est forcément complétée par des connaissances extra-linguistiques.

Le recours aux couleurs pour désigner les contenants des déchets se justifie, en effet, par la volonté de standardiser les couleurs associées aux collectes et d'uniformiser la 
signalisation pour faciliter le tri des matières ${ }^{18}$. Toutefois il n'existe aucune convention européenne qui attribue une couleur donnée à un bac utilisé pour le tri sélectif, les types de déchets par conteneur différant d'un territoire à l'autre, voire d'un pays à l'autre ${ }^{19}$, ce qui empêche de manière dangereuse la mise en place de standards de couleurs au niveau européen. Cela signifie que l'AdjCoul présent dans le syntagme contribue au processus de sous-catégorisation et que ce choix repose plutôt au niveau administratif local que supranational. Comme le fait remarquer Bosredon, il faut en effet que « certaines conditions pragmatico-culturelles se trouvent réalisées » (Bosredon, 1991: 96), et qu'elles soient capables de donner des indications à l'énonciateur vers un sens plutôt que l'autre.

Dans cette perspective, les recherches menées pour notre étude ont fait émerger l'hypothèse qu'un code couleur existe sur le plan pragmatique pour le tri des déchets en vue d'une uniformisation pour tous les pays de l'Union européenne. Des couleurs suffisamment normalisées côtoient des couleurs non normalisées, mais employées de manière systématique dans la pratique du tri sélectif afin de récupérer une quantité élevée de résidus.

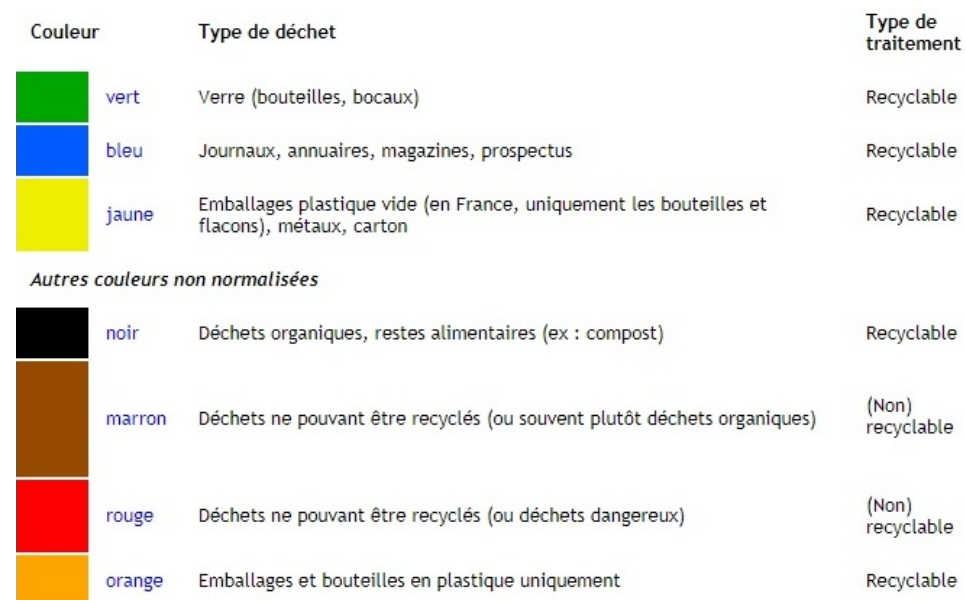

Fig. 3. Code couleur pour désigner de différents types de conteneurs. Source : http://triselectif.e-monsite.com/pages/normes-pour-le-tri-selectif-des-dechets.html

Dans la pratique, le choix des couleurs vise une simplification formelle qui tend à substituer l'emploi d'une terminologie complexe dans le but de rendre la communication avec les citoyens la plus immédiate possible. Sur le plan référentiel, le rouge et le marron semblent être, à notre avis, les deux seules couleurs pour lesquelles la dénomination garde un lien avec les déchets désignés, bien que ce lien ne réside pas dans le chromatisme mais dans certaines conditions pragmatico-culturelles. Le marron renvoie, en effet, aux résidus organiques, tels que les déchets bruns analysés précédemment, et le rouge aux déchets dangereux, le rapport entre la couleur et le produit désigné relevant du caractère symbolique dont le rouge est généralement porteur dans les codes d'alerte.

Le pouvoir évocateur des couleurs sur le plan conceptuel et leur efficacité communicative engendrent la coexistence de plusieurs syntagmes particuliers qui suivent la structure N+AdjCoul, mais dans lesquels l'usage de l'AdjCoul renvoie métonymiquement aux conteneurs pour le tri sélectif plutôt qu'à un trait spécifique des déchets désignés. Bien qu'ils ne soient pas encore très fréquents dans des contextes institutionnels, les ST déchet jaune, déchet rouge et déchet marron existent dans la pratique discursive et désignent par métonymie les déchets contenus dans les bacs auxquels correspond la couleur du ST: 
9) Si séparer ainsi les déchets selon leur nature ne demande pas de temps, ce n'est pas le cas de leur valorisation car les déchets jaunes et verts doivent être amenés auprès de grandes poubelles réservées au tri sélectif (http://life.orlulas.fr/forum/110/4/1).

10) Le transfert doit être interdit et pas uniquement par le biais d'une procédure plus ferme pour les déchets rouges ! (http://context.reverso.net/traduzione/italiano-francese).

Cependant, en ce qui concerne cette dernière catégorie, comme indiqué dans un communiqué de presse de la Commission européenne, le règlement actuel opère une distinction supplémentaire entre les déchets rouges, oranges et verts, en fonction de leur nocivité ${ }^{20}$. Cependant, quant à la couleur verte, la lexicalisation complète du syntagme déchet vert pour les déchets végétaux empêche qu'une métonymie du type contenantcontenu - dans ce cas poubelle verte-verre, puisse exister.

\section{Conclusion}

Dans cette étude, nous avons analysé les adjectifs impliqués dans des ST qui désignent des catégories spécifiques de déchets ayant des propriétés référentielles bien données. S'il est vrai qu'en général le nom est voué à la fonction de dénomination, il en n'est pas le seul responsable: un autre signe, l'adjectif, assume ce rôle. L'analyse des séquences dénominatives du type déchet+Adj nous a amené à constater que la plupart des adjectifs employés relèvent de la langue commune. Il s'agit des adjectifs classifiants et des adjectifs de couleur qui sont susceptibles de devenir catégoriels. Cette valeur hyperonymique se manifeste en discours, entre autres, par leur autonomisation sémantique.

Il en est ainsi pour les ST déchet ordinaire, déchet classique, déchet conventionnel, déchet volumineux, etc. dans lesquels la valeur référentielle du nom s'affaiblit en faveur de la valeur sémantique de l'adjectif qui tend, d'ailleurs, à se substantiver. Des dénominations telles que déchet sauvage ou déchets encombrants désignent donc des éléments de la réalité extra-linguistique, mais elles ne la constituent pas vraiment. La responsabilité linguistique et langagière de la communauté des locuteurs est donc fortement engagée dans l'emploi des adjectifs à valeur dénominative renvoyant à des référents difficilement intelligibles. Dans certains cas, les adjectifs renvoient, souvent de manière métonymique et/ou métaphorique, soit à des propriétés physiques des déchets, soit aux modalités de leur traitement, et acquièrent un rôle capital dans leur catégorisation.

Dans d'autres cas, la fonction descriptive de l'adjectif est moindre, étant donné que celui-ci n'a pas un but descriptif proprement dit, mais il sert à classer les déchets et à les différencier des autres référents. C'est le cas des syntagmes terminologiques impliquant des AdjCoul pour lesquels l'AdjCoul sert à créer des sous-catégories, sans pour autant faire en sorte que le chromatisme prenne le dessus sur des conditions pragmatiques ou plus spécifiquement culturelles.

En effet, nous avons constaté que des facteurs extra-linguistiques entrent en jeu dans le processus de catégorisation des déchets lorsque pour les bacs le recours aux couleurs se justifie notamment par la volonté de standardiser les couleurs associées aux collectes et d'uniformiser la signalisation pour faciliter autant le tri des matières que l'interaction verbale à ce sujet. Le déchet est ainsi défini en fonction de la couleur du bac lui correspondant. 
L'étude sur l'adjectif dans la dénomination des déchets montre que la structure d'un syntagme $\mathrm{N}+$ Adj tire sa valeur de plusieurs dimensions syntagmatiques indissociables, exerçant toutes une influence égale sur le processus de cohésion entre le support nominal et l'adjectif qui composent le syntagme : le caractère prototypique de l'adjectif (Goes 1999); l'histoire et la sémantique de l'adjectif ainsi que la relation qu'il entretient avec le nom (L’Homme 2004) ; la typologie de discours dans lequel l'adjectif est employé ; la place de l'adjectif dans le paradigme et dans le type de discours auquel il appartient.

Ce type d'étude nous mène à la frontière du groupe nominal et du phénomène de la composition par adjectif, autrement dit à la frontière du lexique et de la pragmatique, étant donné que le domaine de spécialité a une forte dimension opérationnelle mais aussi une forte connotation divulgative. La question du classement et de la classifiance semble donc impliquer aussi bien la compétence linguistique et interprétative des locuteurs experts que le contexte discursif des profanes se basant exclusivement sur la valeur sémantique des adjectifs. Dans le traitement et la gestion des déchets, l'adjectif n'entre pas exclusivement dans la constitution de taxinomies car il répond aux exigences de catégorisation mais aussi aux besoins discursifs des locuteurs. Cela détermine parfois un dédoublement dénominatifs (volumineux, encombrant(s)) ou une référentialité faible (déchets blanc ou déchets gris) qui, quoi qu'il en soit, s'autorégularise en discours et, sur le plan normatif, en fonction du discours. En effet, le discours de vulgarisation et le discours spécialisé montrent bien combien l'emploi de l'adjectif peut être complexe sur le plan sémantique (effet de polysémie) et, en même temps, très efficace sur le plan pragmatique.

\section{Références bibliographiques}

Altmanova, J. (2013). Des chiffonniers à la collecte sélective : de la terminologie à la sémiologie. Plaisance, 28, pp. 127-136.

Assal A. (1994). La métaphorisation terminologique. Terminologie et traduction, 2, pp. 235-242.

Bosredon, B. (1991). Prédicativité et lexicalisation. Studia Romanica Posnaniensia, 16, pp. 85-100.

Daille, B. (2001). Qualitative Term Extraction: Identifying relational adjectives. Bourigault, D., Jacquemin, C., L'Homme, M.-C. (éds.). Recent Advances in Computational Terminology. Amsterdam/Philadelphia : John Benjamins, pp. 149-166.

Dixon, R. M. W. (1982). Where Have All the Adjectives Gone? And other Essays in Semantics and Syntax. Berlin-New-York-Amsterdam : Mouton, pp. 1-62.

Dixon, R. M. W. (1977). Where have all the adjectives gone?. Studies in Language, I, pp. 19-80.

Drouin, P. (2003). Term extraction using on-technical corpora as a point of leverage. Terminology, 9 (1), pp. 99-117.

Frassi, P. (2018). L'adjectif et sa définition lexicographique. Berne : Peter Lang.

Galazzi, E., Desoutter, C. (éds.) (2017). Les déchets mis en mots. Paris : Harmattan.

Goes, J. (1999). L'adjectif entre nom et verbe. Bruxelles : Duculot-De Boeck et Larcier.

Gross, G. (1996). Les expressions figées en français. Paris : Ophrys.

Kleiber, G. (1979a). À propos de l'ambiguïté Transparence/Opacité. Travaux de Linguistique et de Littérature, 17, 1, pp. 233-250.

Kleiber, G. (1979b). Problèmes de référence : Descriptions définies et noms propres. Thèse de Doctorat d'État. Université de Strasbourg, parue en 1981, Klincksieck.

Kleiber, G. (2001). Remarques sur la dénomination. Linguistique de la dénomination, Cahiers de praxématique, $36, \mathrm{pp}$. 21-41.

L'Homme, M.-C. (1998). Le statut du verbe en langue de spécialité et sa description lexicographique. Cahiers de lexicologie, 73(2), pp. 61-84.

L'Homme, M.-C. (2004). Adjectifs dérivés sémantiques (ADS) dans la structuration des terminologies. Actes de Terminologie, Ontologie et Représentations des Connaissances. Lyon: Université Jean Moulin Lyon 3 (http://olst.ling.umontreal.ca/pdf/lhomme-lyon2003.pdf). 
Leduc-Adine, J.-R (1980). Polysémie des adjectifs de couleur. Cahiers de lexicologie, 37(2), pp. 6790.

Lerat, P. (2016). Langue et technique. Paris : Hermann.

Maniez, F. (2001a). Ambiguïté syntaxique des groupes nominaux en anglais médical. Cahiers de l'APLIUT, XX (4), pp. 53-65.

Maniez, F. (2001b). Désambiguïsation syntaxique des groupes nominaux en langue spécialisée : le cas des adjectifs en anglais. Maurel, D. (éd.). Actes du colloque TALN de Tours, 2-5 juillet 2001, Tome 1, pp. 273-282.

Maniez, F. (2002). Distinguer les termes des collocations : étude sur corpus du patron <AdjectifNom> en anglais médical. Actes du colloque TALN de Nancy, 24-27 juin 2002, Tome 1, pp. 345350 .

Marengo, S. (2012). Les adjectifs jamais attributs. Syntaxe et sémantique des adjectifs constructeurs de la référence. Bruxelles : De Boek Duculot.

Rakotonoelina, F. (2017). La notion de «déchet(s) »: sémantique dans les discours lexicographiques et configurations discursives sur les sites institutionnels et associatifs français. Galazzi, E., Desoutter, C. (éds.). Les déchets mis en mots. Paris : Harmattan, pp. 15-32.

Rodríguez Pedreira, N. (2002). Recherches sur les adjectifs de couleur. Description et référenciation. L'Information Grammaticale, 95, pp. 27-30.

Zollo, S. D. (2012). La terminologie des déchets dans les discours de spécialité. Mémoire de Master en Linguistique et traduction spécialisée (sous la direction de Jana Altmanova). Napoli : Università degli Studi di Napoli «L'Orientale ».

${ }^{1}<\mathrm{http}: / /$ www.dictionnaire-environnement.com/dictionnaire-dechet.php $>$.

${ }^{2}<$ http://olst.ling.umontreal.ca/?page_id=214>.

${ }^{3}<$ http://www.granddictionnaire.com/ $>$.

${ }^{4}<\mathrm{http}$ ://termeco.info/ecoressources/index.html $>$.

${ }^{5}<$ http://www.culture.fr/franceterme/>.

${ }^{6}<$ http://www.btb.termiumplus.gc.ca/tpv2alpha/alpha-eng.html?lang=eng $>$.

${ }^{7}<$ http://iate.europa.eu $>$.

${ }^{8}<$ www.lerobert.fr $>$.

${ }^{9}<$ http://atilf.atilf.fr/ $>$.

${ }^{10}<\mathrm{http}: / /$ www.ademe.fr/particuliers-eco-citoyens/déchets $>$ (consulté en mai 2017).

$11<$ http://www.preventiondechets.fne.asso.fr> (consulté en mai 2017).

$12<$ http://www.reduisonsnosdechets.fr> (consulté en mai 2017).

${ }^{13}$ La superposition référentielle de ces adjectifs démontre que leur valeur taxinomique est moindre. Signalons également le synonyme monstres présent dans le GDT.

14 Pour la définition complète de déchet inerte, consulter la page suivante : $<$ http://www.dictionnaire-environnement.com/dechet_inerte_ID27.html $>$.

$15<\mathrm{http}: / /$ seod.ch/guide-des-dechets/tri-par-dechet/inertes.html $>$.

${ }^{16}$ Le Dictionnaire de l'Environnement renvoie aussi à l'entrée biodéchet, hyponyme de déchet vert.

${ }^{17}$ Le GDT propose également le synonyme résidu vert et l'adjectif relationnel déchet de jardin comme hyponyme de déchet vert.

${ }^{18} \mathrm{Au}$ Québec la convention pour les couleurs a été adoptée par Recyc-Québec, la société québécoise de récupération et de recyclage, au début des années 2000.

$19<$ https://www.happyloop.fr/poubelles-de-tri-selectif-differents-codes-couleurs-europe/ $>$.

${ }^{20}<$ http://europa.eu/rapid/press-release_IP-95-108_fr.htm $>$. 